\title{
Applicability of BPN and MLP Neural Networks for Classification of Noises Present in Different Image Formats
}

\author{
T. Santhanam \\ Assoc. Prof. \& Head \\ PG \& Research Dept. of Computer Science \\ D. G. Vaishnav College, Chennai, India
}

\author{
S. Radhika \\ Assoc. Prof. \\ Dept. of Computer Applications \\ R. M. K. Engineering College, TN, India
}

\begin{abstract}
Images exist in different formats in real time applications. There is no prescribed format in which an image should be presented as input to any image processing algorithm. This article experiments a neural network approach to classify the noises present in an image given in BMP (Bitmap), JPG/JPEG(Joint Photographic Experts Group), TIF/TIFF(Tagged Image File Format), GIF(Graphics Interchange Format) and PNG(Portable Network Graphics) format. The noises in the image are classified by extracting the statistical features like skewness and kurtosis, which is then applied to the Back Propagation Network (BPN) and Multi Layer Perceptron (MLP). This is done for images of all the formats. MLP is superior in classifying salt and pepper noise in images stored in PNG format. BPN is performing well in classifying Gaussian white noise in images stored in BMP format. The study throws light on the type of neural network to be employed for classifying the different noises present in images of different formats, which will prove to be useful in enhancing the image for further processing.
\end{abstract}

\section{General Terms}

Pattern Classification, Image Processing

\section{Keywords}

Skewness, Kurtosis, Neural Networks, Multi Layer Perceptron, Back Propagation Network, Image formats

\section{INTRODUCTION}

Digital images contain information of significant importance for many research applications like satellite television, computer tomography, magnetic resonance imaging etc. [1]. The images used by these applications should be of high quality to yield superior results.

Denoising these images plays a crucial role in enhancing the image for further processing. Luo used a fuzzy approach for the efficient removal of impulse noise from the digital images. [2] Abreu et al. have designed a framework using Rank ordered mean filter for the removal of noise from highly corrupted images. [3] A statistical model using Bayesian least squares estimator is used for image denoising by Hammond and Simoncelli. [4] Noise is also removed from image data using recursive neuro fuzzy filters [5]

Research in the past has revealed that automated techniques for the identification of the noise is of higher significance in the recent times and Motwani and Harris have discussed many denoising approaches. [6] Chehdi and Sabri have defined the identification criteria based on the analysis of local variations of the average and the standard deviation of the observed image. [7]

Beaurepaire et al, have characterized each class of noise by a parameter obtained from histograms computed on several homogeneous regions of the observed image. [8] The homogeneous regions are obtained by segmenting images. Then, the estimation of the standard deviation is achieved from the analysis of a histogram of local standard deviations computed on each of the homogeneous regions. Vozel et al. has proposed an unsupervised variational classification through a multi thresholding method. [9] Chen and Das have proposed a simple pattern classification based noise identification by making use of the statistical features. [10] Santhanam and Radhika have used a neural network based approach to classify the noises. [11]

The main objective of this research was to conduct a comparative study among the BPN and MLP neural network models to classify the Gaussian white, non Gaussian white and salt and pepper noise present in the images stored in different formats like BMP, JPG, TIFF, GIF and PNG. The result of this classification leads to efficient denoising, that is a preliminary process of other automated image processing operations.

\subsection{Image formats}

Images are stored in different formats based on the applications in which they are used. BMP is a common image format in Windows Applications. It offers millions of colors and there is no loss in the quality of the image stored. But it results in large file size.

JPG is the format for photo images used in a digital camera. JPG modifies the image pixel data to be more convenient for compression. The compressed form results in lossy compression. The lost data is always unrecoverable. But this is compromised with the reduction in file size which helps in transfer of web pages and email files that needs to be small, when transferred via modem.

TIFF is recognized by various computers and is suitable for text and black and white images. It is the standard format for scanned documents and Optical Character Recognition. It makes use of lossless compression which results in high quality of image, but with a larger file size which consumes memory storage.

GIF is apt for storing graphics with a limited number of colours such as clip art, drawings, text, diagrams and small pictures. The 
advantage of this format is that it supports animation and is largely used on the web to give special effects to the contents in the web pages. The drawback is that the maximum number of colours is limited to 256 so that some images will not look good in this format.

PNG is used by the internet community in the recent times. It allows users to save, change and resave the file without loss of quality. But it doesn't support animations and also not compatible with all browsers.

\subsection{Artificial Neural Networks (ANN)}

An ANN is a powerful data-modeling tool to capture and represent complex input/output relationships. The motivation for the development of neural network technology stemmed from the desire to develop an artificial system that could perform "intelligent" tasks similar to those performed by the human present in the images stored in BMP, JPG, TIFF, GIF and PNG format is also tabulated.

\subsubsection{Back Propagation Network (BPN)}

Back propagation is a form of supervised learning for multilayer nets, also known as the generalized delta rule. Error data at the output layer is "back propagated" to earlier ones, allowing incoming weights to these layers to be updated. It is most often used as training algorithm in current neural network applications. The back propagation algorithm was developed by Paul Werbos [14] and then, Rumelhart et al [15] and Parker [16] worked on it. From then onwards, the back propagation algorithm has been widely used as a learning algorithm in feed forward multilayer neural networks. BPN is widely used for classification problems. Kumaravel and Reddy [17] have used BPN for classification of bone samples at different locations of the jawbone region and Ibrahim et al have used it to classify document zone content in technical document images. [18] Khanale and Chitnis have used BPN for classifying and recognising handwritten devanagiri characters. [19] Coban has employed BPN to identify the genotypes of grapevine and has concluded that the network handles the unknown variety with certainty. [20] Md Saad et al used the BPN to classify the heart abnormalities by utilising the features from Time frequency Spectogram. [21] The BPN architecture is shown in Figure 1.

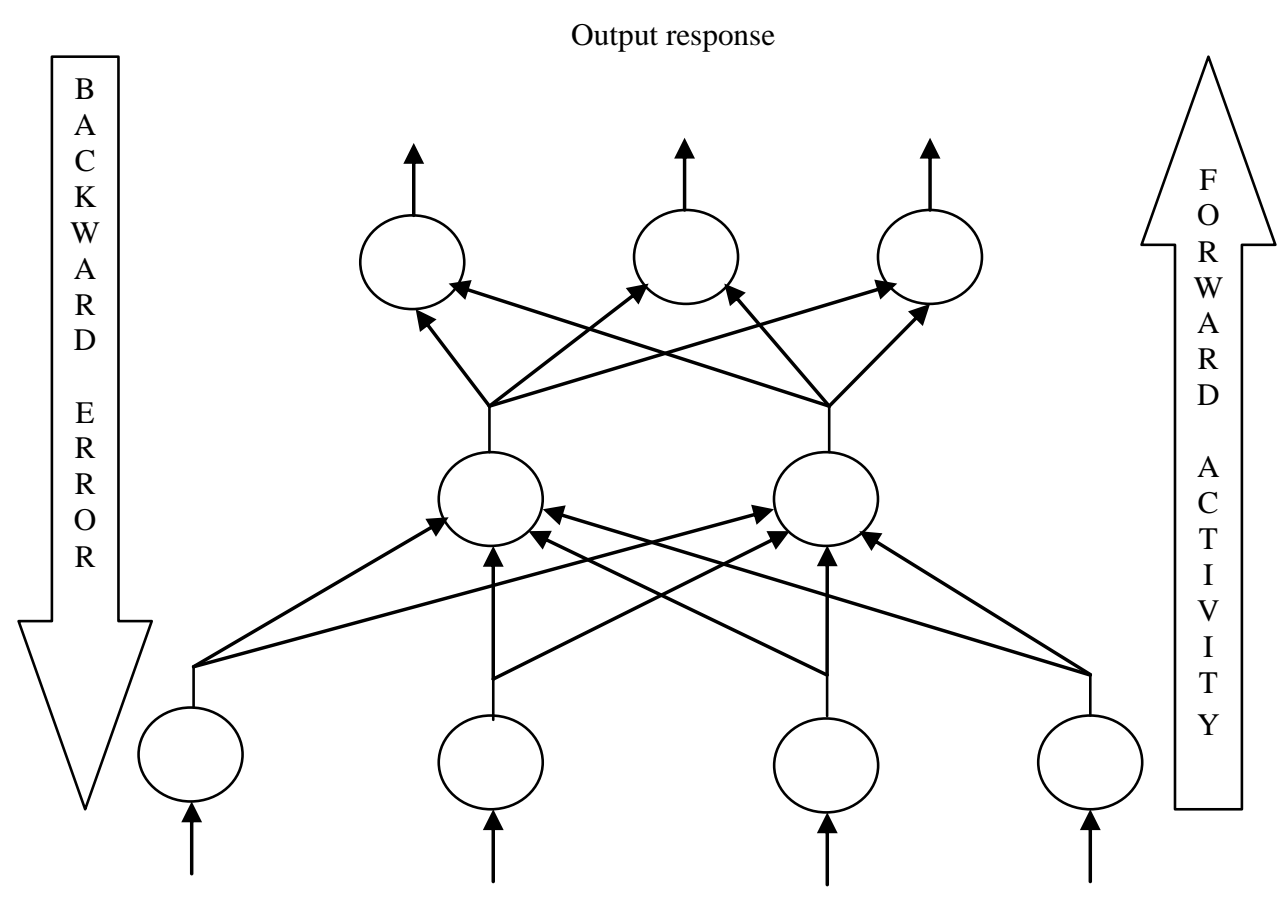

Input pattern

\section{Figure 1: Architecture of BPN}

brain. Senol and Ozturan suggested an ANN approach for prediciting the Stock Price behavior. [12] Further, Eriki and Udegbunam used an ANN for evaluating the prices of housing units in Nigeria. [13] In this paper, the performance analysis of Back Propagation Network (BPN) and Multi Layer Perceptron (MLP) is discussed. The results of the correctly classified noise
The process starts by applying the first input pattern and the corresponding target output. The input causes a response to the neurons of the first layer, which in turn cause a response to the neurons of the next layer, and so on, until a response is obtained at the output layer. That response is then compared with the target response; and the difference (the error signal) is calculated. From the error difference at the output neurons, the algorithm computes the rate at which the error changes as the 
activity level of the neuron changes. So far, the calculations were computed forward (i.e., from the input layer to the output layer).

Now, the algorithm steps back one layer before that output layer and recalculate the weights of the output layer (the weights between the last hidden layer and the neurons of the output layer) so that the output error is minimized. The algorithm next calculates the error output at the last hidden layer and computes new values for its weights (the weights between the last and next-to-last hidden layers). The algorithm continues calculating the error and computing new weight values, moving layer by layer backward, toward the input.

When the input is reached and the weights do not change, (i.e., when they have reached a steady state), then the algorithm selects the next pair of input-target patterns and repeats the process. Although responses move in a forward direction, weights are calculated by moving backward, hence the name back propagation.

\subsubsection{Multi Layer Perceptron (MLP)}

An MLP is a network of simple neurons called perceptrons. It is a feedforward neural network with one or more layers between input and output layer. Feedforward means that data flows in one direction from input to output layer. MLPs are widely used for pattern classification, recognition, prediction and approximation. Foody [22] have used MLP to classify remote sensed data of an agricultural site and Antani et al. [23] have used it for vertebra shape classification. The MLP architecture is shown in Figure 2

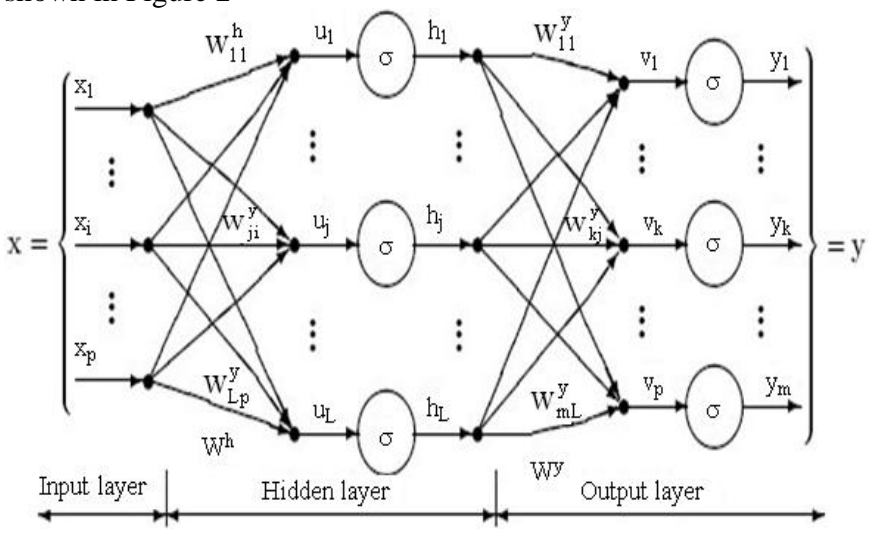

Figure 2 Architecture of a MLP

This network has an input layer (on the left) with three neurons, one hidden layer (in the middle) with three neurons and an output layer (on the right) with three neurons.

There is one neuron in the input layer for each predictor variable. In the case of categorical variables, $N$-1 neurons are used to represent the $N$ categories of the variable.

Input Layer - A vector of predictor variable values $\left(x_{I} \ldots x_{p}\right)$ is presented to the input layer. The input layer distributes the values to each of the neurons in the hidden layer.

Hidden Layer - Arriving at a neuron in the hidden layer, the value from each input neuron is multiplied by a weight $\left(w_{j i}\right)$, and the resulting weighted values are added together producing a combined value $u_{j}$. The weighted sum $\left(u_{j}\right)$ is fed into a transfer function, $\sigma$, which outputs a value $h_{j}$. The outputs from the hidden layer are distributed to the output layer.

Output Layer - Arriving at a neuron in the output layer, the value from each hidden layer neuron is multiplied by a weight $\left(w_{k j}\right)$, and the resulting weighted values are added together producing a combined value $v_{j}$. The weighted sum $\left(v_{j}\right)$ is fed into a transfer function, $\sigma$, which outputs a value $y_{k}$. The $y$ values are the outputs of the network.

\section{MATERIALS AND METHODS}

\subsection{Noise Identification}

Image noise is ubiquitous despite sophisticated image capturing techniques. There are different types of image noise, viz. Gaussian noise, salt and pepper noise, speckle noise, shot noise and quantization noise. Understanding the noise characteristics helps in the classification of noise, so that appropriate filters can be used to enhance the quality of image. Skewness is the opposite of symmetry. i.e. pixels that is darker or lighter than the mean. Kurtosis is a measure of peakedness. It represents for a given variance, the over representation or under representation of frequencies in the middle of the range compared to normal distribution. The use of these statistical measures helps in classifying the noises. They are measured as given in equations (1) and (2).

Skewness is the standardized third central moment of the probability distribution. Skewness is a measure of symmetry, or more precisely, the lack of symmetry. A distribution, or data set, is symmetric if it looks the same to the left and right of the center point.

$S=\frac{E(X-\mu)^{3}}{\sigma^{3}}$

Kurtosis is the standardized fourth central moment of the probability distribution. Kurtosis is a measure of whether the data are peaked or flat relative to a normal distribution. That is, data sets with high kurtosis tend to have a distinct peak near the mean, decline rather rapidly, and have heavy tails. Data sets with low kurtosis tend to have a flat top near the mean rather than a sharp peak. A uniform distribution would be the extreme case.

$S=\frac{E(x-\mu)^{4}}{\sigma^{4}}-3$

where the (-3) term makes the value zero for a normal distribution.

\subsection{Implementation}

The noises that are classified in this proposed technique are Non Gaussian white, Gaussian white and salt and pepper. These noises are additive in nature. When gaussian noise is present in an image, each pixel in the image is changed from its original value by a small amount and in case of salt and pepper noise's presence in an image; pixels in the image are very different in color or intensity from their surrounding pixels. The steps 
involved in the methodology of noise classification are summarized as follows:

1. Get an image in a particular format(say BMP)

2. Introduce the noises. (i.e.) salt \& pepper, non Gaussian white and Gaussian white.

3. Get the noise samples by filtering salt \& pepper noise using median filter and gaussian noise using wiener filter.

4. Estimate the skewness and kurtosis of the noise samples.

5. The values of skewness and kurtosis are given as inputs to the BPN and MLP network. At random, distribute the images to $\mathrm{K}$ partitions of equal sizes (say 10). Training is carried out with $(\mathrm{K}-1)$ partitions. $(\mathrm{k}-$ folding technique)

6. Then, testing is performed with the left out partition.

7. The above process is repeated $\mathrm{k}$ times and during each cycle ( $\mathrm{i}^{\text {th }}$ cycle), $\mathrm{i}^{\text {th }}$ partition is used for testing and the rest of the partitions are temporarily combined for training the network and the resulting accuracy is recorded.

8. Lastly, the result of each run is added and final average is computed to determine the resulting accuracy.

The steps are repeated for JPG, GIF, TIFF and PNG formats and the results are tabulated as given below.

The CASIA-Irisv3 database has been used to test the proposed technique. (http://www.sinobimetrics.com) The database contains about 4500 iris images of which 250 left eyes and 250 right eye images were selected at random resulting in 500 images. Then, the three different types of noises were introduced with intensity as 0.02 , making the mugshot database to 1500 images. i.e. (500 images with Non-Gaussian white noise, 500 images with Gaussian white noise and the rest 500 with salt and pepper noise). Online image converter software was used to convert the images in to various formats. The experiment was performed using the MATLAB tool.

\subsection{Findings}

The values in Table 1 indicate the percentage of images classified correctly by the BPN and MLP network for the images given in JPG, BMP, PNG, GIF and TIFF formats. BPN is showing a classification accuracy of $90 \%$ classifying non Gaussian White noise and salt and pepper noise in images stored in GIF format and an accuracy of an average of $94 \%$ in classifying gaussian white noise in images stored in JPG, BMP and GIF format. Whereas, MLP exhibits a superior performance in classifying non gaussian white noise $(96.6 \%)$ and salt and pepper noise $(98.92 \%)$ in images stored in TIFF and PNG format respectively. Further, MLP scores better than BPN in classifying non Gaussian White noise in images stored in JPG, BMP, PNG and TIFF format.

\section{CONCLUSION}

The article discusses about the performance of BPN and MLP neural networks in classifying the various noises like Nongaussian white, Gaussian white and salt and pepper noise present in images stored in various formats like JPG, BMP,
TIFF, PNG and GIF. The scope of the future work is to find out a neural network to yield consistent performance to detect the noises present in the images of any format. The classification accuracy of both networks employed in this study is satisfactory with BPN giving an average accuracy of $88.23 \%$ and that of MLP is $90.93 \%$. From this study, one can easily conclude the type of neural network to be considered for detecting the various kinds of noises present and as well for a particular image format.

\section{REFERENCES}

http://www.codeding.com/?article $=10$

[2] Luo, W., 2006. Efficient Removal of Impulse Noise from Digital Images. IEEE Transaction on Consumer Electronics, vol. 52, pp. 523-527

[3] Abreu, E., M. Lightstone, S.K. Mitra and K. Arakawa, 1996. A new efficient approach for the removal of impulse noise from highly corrupted images. IEEE Trans. Image Process., 5: 1012-1025

[4] Hammond, D.K. and E.P. Simoncelli, 2006. Image denoising with an orientation-adaptive gaussian scale mixture model. Proceedings of the IEEE International Conference on Image Processing, Oct. 8-11, Atlanta, GA., pp: 1433-1436

[5] Russo, F., 2000. Noise removal from image data using recursive neurofuzzy filters. IEEE Trans. Instrument. Measure., 49: 307-314

[6] Motwani, M.C. and F.C. Harris, 2004. Survey of image denoising techniques. Proceedings of the GSPx, Sept. 2730, Santa Clara, CA., pp: 27-30.

[7] Chehdi, K. and M. Sabri, 1992. A new approach to identify the nature of the noise affecting an image. Proc. IEEE Int. Conf. Acoustics, Speech Signal Process., 3: 285-288.

[8] Beaurepaire, L., K. Chehdi and B. Vozel, 1997. Identification of the nature of noise and estimation of its statistical parameters by analysis of local histograms. Proc. IEEE Int. Conf. Acoustics, Speech Signal Process., 4: 2805-2808

[9] Vozel, B., K. Chehdi, L. Klaine, V.V. Lukin and S.K. Abramov, 2006. Noise identification and estimation of its statistical parameters by using unsupervised variational classification. Proceedings of the IEEE International Conference on Acoustics, Speech and Signal Processing, May 14-19, IEEE Xplore Press, Toulouse, pp: 841-844.

[10] Chen, Y. and M. Das, 2007. An automated technique for image noise identification using a simple pattern classification approach. Proceedings of the MWSCAS, (MWSCAS'07), IEEE Computer Society, USA., pp: 819822.

[11] Santhanam, T. and S. Radhika, 2010. A novel approach to classify noises in images using artificial neural network. Journal of Computer Science 6 (5): pp. 506 -510.

[12] Senol, D. and M. Ozturan, 2008. Stock price direction prediction using artificial neural network approach: The case ofTurkey.J.Artif.Intell.,1:70-77. 
[13] Eriki, P.O. and R.I. Udegbunam, 2008. Application of neural network in evaluating prices of housing units in Nigeria:Apreliminaryinvestigation.J.Artif.Intell.,1:21-27.

[14] Werbos, P., 1974. Beyond regression: New tools for prediction and analysis in the behavioral sciences. Ph.D. Thesis, Harvard University, Cambridge, Mass.

[15] Rumelhart, D. E., J.L. McClelland and the PDP Research Group, 1986. Parallel Distributed Processing Exploration in the Micro Structure of Cognition.. Vol. 1,. MIT Press, Cambridge, MA, pp: 547.

[16] Parker, D., 1982. Learning logic. Invention Report S81-64, File 1, Office of Technology Licensing, Stanford University.

[17] Kumaravel, N. and T.K. Reddy, 2009. Texture Analysis of Bone CT Images for Classification and Characterization of Bone Quality", International Journal of Soft Computing 4(5), pp. 223-228.

[18] Ibrahim, Z., D. Isa, R. Rajkumar and G. Kendall, 2009. Document zone content classification for technical document images using artificial neural networks and support vector machines. Proceedings of the 2nd International Conference on the Applications of Digital Information and Web Technologies, Aug. 4-6, London, pp: 345-350.

[19] Khanale, P.B. and S.D. Chitnis, 2011. Handwritten Devanagiri Character Recognition using Artificial Neural Network. Journal of Artificial Intelligence 4(1) pp 55-62.

[20]Coban, H., 2004. Application of an Artificial Neural Network (ANN) for the identification of grapevine (Vitis viniferaL.)genotypes.AsianJ.PlantSci.,3:340-343.

[21] Md Saad, M.H., M.J. Mohd Nor, F.R.A. Bustami and R. Ngadiran, 2007. Classification of heart abnormalities using artificialneuralnetwork.J.AppliedSci.,7:820-825.

[22] Foody, G.M., 2001. Thematic mapping from remotely sensed data with neural networks: MLP, RBF and PNN based approaches. J. Geogr. Syst., 3: 217-232.

[23] Antani, A., L.R. Long, G.R. Thoma and R.J. Stanley, 2003. Vertebra shape classification using MLP for content-based image retrieval. Proc. Int. Joint Conf. Neural Networks, 1: $160-165$.

Table 1. Comparative results of BPN and MLP for images in various format

\begin{tabular}{|c|c|c|c|c|c|c|c|c|c|c|}
\hline Format & \multicolumn{2}{|c|}{$J P G$} & \multicolumn{2}{|c|}{ BMP } & \multicolumn{2}{|c|}{$P N G$} & \multicolumn{2}{|c|}{ GIF } & \multicolumn{2}{|c|}{ TIFF } \\
\hline \begin{tabular}{|l|}
$\begin{array}{c}\text { Neural } \\
\text { model }\end{array}$ \\
Noise
\end{tabular} & $\begin{array}{l}\text { BPN } \\
(\%)\end{array}$ & $\begin{array}{l}\text { MLP } \\
(\%)\end{array}$ & $\begin{array}{l}\text { BPN } \\
(\%)\end{array}$ & $\begin{array}{l}\text { MLP } \\
(\%)\end{array}$ & $\begin{array}{l}\text { BPN } \\
(\%)\end{array}$ & $\begin{array}{l}\text { MLP } \\
(\%)\end{array}$ & $\begin{array}{l}\text { BPN } \\
(\%)\end{array}$ & $\begin{array}{l}\text { MLP } \\
(\%)\end{array}$ & $\begin{array}{l}\text { BPN } \\
(\%)\end{array}$ & $\begin{array}{l}\text { MLP } \\
(\%)\end{array}$ \\
\hline $\begin{array}{c}\text { Nongaussia } \\
n \text { white }\end{array}$ & 81.80 & 88.66 & 82.41 & 89.32 & 78.13 & 93.14 & 90.38 & 92.31 & 88.42 & 96.60 \\
\hline $\begin{array}{c}\text { Gaussian } \\
\text { white }\end{array}$ & 94.00 & 93.41 & 95.40 & 94.68 & 85.98 & 88.57 & 93.27 & 86.54 & 84.76 & 89.72 \\
\hline $\begin{array}{c}\text { Salt \& } \\
\text { Pepper noise }\end{array}$ & 89.11 & 83.04 & 88.57 & 84.47 & 89.69 & 98.92 & 93.48 & 90.22 & 88.00 & 93.33 \\
\hline
\end{tabular}

